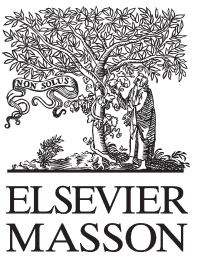

Disponible en ligne sur

Elsevier Masson France

ScienceDirect

www.sciencedirect.com
EM|consulte

www.em-consulte.com
Médecine

Nucléaire

Imagerie Fonctionnelle et Métabolique

Médecine Nucléaire xxx (2015) xxx-xxx

Mise au point

\title{
Apport de la tomographie par émission de positons (TEP) cérébrale dans les encéphalites dysimmunitaires
}

\section{Interest of cerebral positron emission tomography (PET) in dysimmune encephalitis}

$$
\text { S. Lagarde }{ }^{a, *}, \text { E. Guedj }{ }^{b}
$$

${ }^{\text {a }}$ Service de neurophysiologie clinique, pôle de neurosciences cliniques, CHU de La Timone, AP-HM, 264, rue Saint-Pierre, 13385 Marseille, France
b Service de médecine nucléaire, CHU de La Timone, AP-HM, 264, rue Saint-Pierre, 13385 Marseille, France

Reçu le 3 mars 2015 ; accepté le 5 mars 2015

\section{Résumé}

Les encéphalites dysimmunitaires sont des pathologies fréquentes et pourvoyeuses de handicap, pour lesquelles il existe des traitements efficaces (immunothérapies). Certains signes cliniques sont évocateurs : évolution rapidement progressive, crise d'épilepsie, mouvements anormaux, troubles psychiatriques, troubles mnésiques, dysautonomie, hyponatrémie. Les examens classiques, notamment l'imagerie par résonance magnétique (IRM) et les analyses standard du liquide céphalo-rachidien (LCR), peuvent être pris en défaut. Dans ce contexte, la tomographie par émission de positons (TEP) cérébrale a un grand intérêt pour le diagnostic. Les patterns retrouvés sont : association d'hypo- et d'hypermétabolisme, gradient hypermétabolisme fronto-temporal/hypométabolisme pariéto-occipital (encéphalites anti- $N$-methyl-D-aspartate receptor [NMDAR]), hypermétabolisme temporal et des ganglions de la base (encéphalite anti-leucine-rich glioma inactivated protein 1 [LGI1]), hypermétabolisme temporal (autres encéphalites limbiques), hypométabolismes pariéto-occipitaux \pm des ganglions de la base (neurolupus), hypométabolisme unihémisphérique diffus (encéphalite de Rasmussen). La confirmation diagnostique pourra nécessiter la recherche d'anticorps spécifique (sérum/LCR). La TEP cérébrale est aussi utile au cours du suivi : efficacité du traitement (amélioration du métabolisme), rechute (aggravation des altérations du métabolisme) et évaluation d'éventuelles séquelles.

Mots clés : Tomographie par émission de positons ; TEP ; Encéphalite ; Paranéoplasique ; Inflammatoire

\begin{abstract}
Dysimmune encephalitis are frequent and disabling pathologies with efficacious treatments (immunotherapy). Evocative clinical signs are: rapid progressive evolution, seizure, movement disorder, psychiatric troubles, memory defect, dysautonomia and hyponatremia. Classical ancillary test, magnetic resonance imaging (MRI) and standard cerebro-spinal fluid (CSF) analysis can be normal. In this context, the cerebral positron emission tomography (PET) is helpful for the diagnosis. More often reported patterns are: association of hyper- and hypo-metabolism; frontotemporal hyper-metabolism/parieto-occipital hypo-metabolism gradient (anti-N-methyl-D-aspartate receptor [NMDAR] encephalitis); temporal and basal ganglia hyper-metabolism (anti-leucine-rich glioma inactivated protein 1 [LGI1] encephalitis); temporal hyper-metabolism (other limbic encephalitis); parieto-occipital \pm basal ganglia hypo-metabolism (neurolupus); uni-hemispheric diffuse hypo-metabolism (Rasmussen encephalitis). The diagnosis could require research of specific antibodies. Finally, the cerebral PET is also useful during the follow-up: treatment's efficacy (improvement of metabolism), relapse (aggravation of metabolism's alteration), and evaluation of possible sequelae.
\end{abstract}

Keywords: Positron emission tomography; PET; Encephalitis; Paraneoplastic; Inflammatory

\footnotetext{
* Auteur correspondant.

Adresse e-mail : stanislas.lagarde@ap-hm.fr (S. Lagarde).
} 


\section{Introduction}

Les encéphalites dysimmunitaires sont des pathologies fréquentes et pourvoyeuses de handicap. Leur spectre s'est élargi avec la description récente d'encéphalites avec anticorps à épitopes intracellulaires (anti-Hu, Ri, Yo, CV2/CRMP5, Ma2, dont synaptique : anti-GAD 65 , amphiphysin) et à épitopes extracellulaires/membranaires (anti-NMDAR, LGI1, $\gamma$-aminobutyric acid-B receptor [GABABR], GABAAR, $\alpha$-amino-3hydroxy-5-methyl-4-isoxazolepropionic acid receptor [AMPAR], contactin-associated protein-like 2 [CASPR2], dipeptidyl-peptidase-like protein-6 [DPPX], metabotropic glutamate receptor 5 [mGluR5]).

Ces encéphalites peuvent être paranéoplasiques rendant leur diagnostic plus aisé en cas de tumeur connue. Au contraire, en l'absence de tumeur, un tableau neurologique aspécifique ou atypique avec des examens paracliniques initiaux normaux rend leur diagnostic difficile. Certaines immunothérapies étant efficaces, un diagnostic rapide et précis est requis. Dans ce cadre, la tomographie par émission de positons (TEP) cérébrale a un rôle important dans la démarche diagnostique et thérapeutique.

Nous exposerons ici quelques exemples d'encéphalites pour lesquelles la TEP cérébrale a un intérêt particulier : encéphalites auto-immunes, neurolupus et encéphalite de Rasmussen.

\section{Encéphalites auto-immunes}

Le point commun clinique aux encéphalites auto-immunes est leur apparition/évolution rapidement progressive. Il existe d'autres éléments évocateurs : mouvements anormaux, hyponatrémie, troubles mnésiques, crise d'épilepsie [1].

Il existe deux grands tableaux cliniques : les encéphalites à anticorps anti-NMDAR et les encéphalites limbiques (EL) [2].

\subsection{Les encéphalites à anticorps anti-NMDAR}

Elles concernent préférentiellement l'enfant et la femme jeune mais touchent tous les âges. Le tableau clinique associe : crise d'épilepsie, troubles psychiatriques (hallucinations, troubles du comportement, catatonie), mouvements anormaux (dystonie notamment orofaciale), troubles cognitifs, dysautonomie, somnolence, hypoventilation. L'évolution peut être sévère et conduire à une prise en charge en réanimation. Elles peuvent être associées à un tératome ovarien, mais avant l'âge de 12 ans les formes paranéoplasiques sont rares [3-6].

\subsection{Les encéphalites limbiques}

Elles proviennent d'une atteinte du système limbique associant troubles mnésiques, confusion mentale et crises d'épilepsies focales temporales, parfois associées à des troubles du langage, du comportement, des hallucinations [2]. Les anticorps impliqués sont : anti-LGI (spasmes brachio-faciaux, Q2 hyponatrémie) [7], anti-Caspr 2 (syndrome de Morvan : EL + crampes/fasciculations) [8], anti-AMPAR (tableau « pseudo-psychiatrique ») [1], anti-Gly (encéphalomyélite avec rigidité et myoclonus) [1], anti-GABA $\mathrm{B}_{\mathrm{B}}$ [1], antiGAD (épilepsie temporale, syndrome de Stiff-Person ou cérébelleux) [9], anti-Hu (encéphalopathie plus diffuse, voire encéphalomyélite) [10], anti-CV2 (atteinte diencéphalique : somnolence, hyperthermie et/ou du tronc cérébral : paralysie de la verticalité du regard, dysarthrie, dysphagie, paralysie faciale) [10], anti-Ma2 (atteinte hypothalamique : somnolence, narcolepsie, cataplexie, hyperphagie et/ou du tronc cérébral) [10], anti-amphiphysin.

Le bilan initial doit rechercher des étiologies infectieuse, métabolique ou toxique. L'existence d'hypersignaux IRM en T2/FLAIR et d'anomalie du LCR (hyperprotéinorachie, pleiocytose, voire bandes oligoclonales) sont évocatrices d'une étiologie auto-immune. Néanmoins, ce bilan peut être pris en défaut $(75 \%$ d'analyses standard du LCR et $50 \%$ d'IRM sont normales) d'où l'utilité de la TEP cérébrale (parfois seul examen anormal).

L'aspect TEP le plus évocateur est l'association d'hyper- et d'hypométabolismes, mais des tableaux avec hypermétabolisme ou hypométabolisme isolé sont possibles. Les altérations peuvent être unilatérales ou bilatérales (souvent asymétriques), et d'étendue variable (corrélée à la sévérité clinique). Dans les encéphalites avec anticorps anti-NMDAR, l'aspect le plus évocateur est l'existence d'un gradient métabolique cortical antéro-postérieur avec hypermétabolisme frontotemporal et hypométabolisme pariéto-occipital. Un hypermétabolisme des ganglions de la base est possible [11-15]. Dans les encéphalites avec anticorps anti-LGI1, on retrouve plus souvent un tableau d'hypermétabolisme touchant les régions temporales et les ganglions de la base. L'association hyper- et hypométabolisme est plus rare et aucun hypométabolisme seul n'a été décrit [11,16,17]. Dans les autres cas publiés d'encéphalite limbique, on retrouve plutôt des hypermétabolismes temporaux [18-20].

De manière générale devant des altérations du métabolisme, le diagnostic d'encéphalite auto-immune ne pourra être retenu que devant un tableau clinique compatible et après la détection d'anticorps spécifique (LCR/sérum ; « à adresser au centre de référence des syndromes neurologiques paranéoplasiques »). Enfin, la TEP cérébrale est utile au cours du suivi : efficacité du traitement (amélioration du métabolisme), rechute (aggravation des altérations du métabolisme) et évaluation d'éventuelles séquelles [21].

Les encéphalites avec anticorps à épitopes intracellulaires sont plus souvent paranéoplasiques, répondent mal aux immunothérapies, et ont un mauvais pronostic fonctionnel $[9,10]$. Les encéphalites avec anticorps à épitopes de surface sont moins souvent paranéoplasiques et répondent mieux aux immunothérapies $[1,8]$. Le bilan à la recherche d'une néoplasie associée doit être systématique, répété [22]. Les tumeurs les plus fréquentes sont pulmonaire (anti-Hu, Cv2, Ma2, AMPA), testiculaire (Ma2, LGI1), thymome (Caspr2, AMPA), tératome ovarien (anti-NMDAR).

La prise en charge repose sur le traitement oncologique et l'utilisation d'immunothérapies (corticostéroïdes, immunoglobulines intraveineuses, plasmaphérèse, cyclophosphamide, rituximab) [2]. 


\section{Neurolupus}

Les atteintes du système nerveux central associées au lupus érythémateux disséminé ne sont pas rares (14-90\% selon les études). Les manifestations cliniques peuvent être : infarctus cérébraux, crise d'épilepsie, mouvements anormaux (chorée), troubles cognitifs, troubles psychiatriques, myélite, méningite aseptique ou céphalée. En dehors des infarctus cérébraux, le diagnostic de l'atteinte neurologique est difficile car l'analyse du LCR et l'IRM montrent des anomalies modérées, aspécifiques, voire l'absence d'anomalie. Dans ce contexte, la TEP a tout son intérêt montrant des anomalies du métabolisme dans environ $75 \%$ des cas avec IRM normale [23]. Il s'agit essentiellement d'hypométabolismes corticaux, préférentiellement pariétaux et occipitaux [24]. À noter que, plus rarement, il peut exister l'association d'hyper- et d'hypométabolisme ou un hypermétabolisme des ganglions de la base [23]. Le traitement du neurolupus se fait selon 3 axes : prévention des infarctus, immunothérapie et traitement symptomatique [25].

\section{Encéphalite de Rasmussen}

L'encéphalite de Rasmussen est une atteinte inflammatoire touchant un seul hémisphère cérébral. C'est une pathologie rare et sévère qui est caractérisée par une épilepsie focale pharmacorésistante, des déficits corticaux progressifs (hémiparésie, déclin cognitif, hémianopsie, trouble phasique) et une atrophie unihémisphérique.

Dans l'encéphalite de Rasmussen, la TEP est un bon complément de l'IRM afin d'arriver à un diagnostic sûr et rapide. La TEP révèle un hypométabolisme unihémisphérique diffus, bien corrélé à l'atrophie visualisée sur l'IRM. Il peut exister des foci hypermétaboliques en cas d'activité épileptique importante [26]. Les traitements antiépileptiques ont toujours une efficacité limitée. Seule l'hémisphérotomie améliore l'épilepsie, mais avec des séquelles fonctionnelles. Dans ce contexte, l'utilisation d'immunothérapies a été rapportée récemment [27].

\section{Conclusion}

Le spectre des encéphalites dysimmunitaires s'est étendu récemment. Il s'agit de pathologies invalidantes pour lesquelles existent des traitements efficaces. Les examens paracliniques classiques peuvent être pris en défaut, la TEP cérébrale a donc toute sa place dans leur prise en charge diagnostique et thérapeutique $[1,9,10,28-30]$.

\section{Déclaration d'intérêts}

Les auteurs déclarent ne pas avoir de conflits d'intérêts en relation avec cet article.

\section{Références}

[1] Irani SR, Gelfand JM, Al-Diwani A, Vincent A. Cell-surface central nervous system autoantibodies: clinical relevance and emerging paradigms. Ann Neurol 2014;76:168-84.
[2] Armangue T, Leypoldt F, Dalmau J. Autoimmune encephalitis as differential diagnosis of infectious encephalitis. Curr Opin Neurol 2014;27: $361-8$.

[3] Titulaer MJ, McCracken L, Gabilondo I, et al. Treatment and prognostic factors for long-term outcome in patients with anti-NMDA receptor encephalitis: an observational cohort study. Lancet Neurol 2013;12: 157-65.

[4] Dalmau J, Lancaster E, Martinez-Hernandez E, Rosenfeld MR, BaliceGordon R. Clinical experience and laboratory investigations in patients with anti-NMDAR encephalitis. Lancet Neurol 2011;10:63-74.

[5] Florance NR, Davis RL, Lam C, et al. Anti-N-methyl-D-aspartate receptor (NMDAR) encephalitis in children and adolescents. Ann Neurol 2009;66: $11-8$.

[6] Dalmau J, Gleichman AJ, Hughes EG, et al. Anti-NMDA-receptor encephalitis: case series and analysis of the effects of antibodies. Lancet Neurol 2008;7:1091-8.

[7] Irani SR, Stagg CJ, Schott JM, et al. Faciobrachial dystonic seizures: the influence of immunotherapy on seizure control and prevention of cognitive impairment in a broadening phenotype. Brain 2013;136:3151-62.

[8] Irani SR, Alexander S, Waters $\mathrm{P}$, et al. Antibodies to Kv1 potassium channel-complex proteins leucine-rich, glioma inactivated 1 protein and contactin-associated protein-2 in limbic encephalitis, Morvan's syndrome and acquired neuromyotonia. Brain 2010;133:2734-48.

[9] Saiz A, Blanco Y, Sabater L, et al. Spectrum of neurological syndromes associated with glutamic acid decarboxylase antibodies: diagnostic clues for this association. Brain 2008;131:2553-63.

[10] Dalmau J, Rosenfeld MR. Paraneoplastic syndromes of the CNS. Lancet Neurol 2008;7:327-40.

[11] Wegner F, Wilke F, Raab P, et al. Anti-leucine rich glioma inactivated 1 protein and anti-N-methyl-D-aspartate receptor encephalitis show distinct patterns of brain glucose metabolism in 18F-fluoro-2-deoxy-d-glucose positron emission tomography. BMC Neurol 2014;14:136.

[12] Lee EM, Kang JK, Oh JS, Kim JS, Shin YW, Kim CY. 18F-Fluorodeoxyglucose positron-emission tomography findings with anti-N-methyl-Daspartate receptor encephalitis that showed variable degrees of catatonia: three cases report. J Epilepsy Res 2014;4:69-73.

[13] Leypoldt F, Buchert R, Kleiter I, et al. Fluorodeoxyglucose positron emission tomography in anti-N-methyl-D-aspartate receptor encephalitis: distinct pattern of disease. J Neurol Neurosurg Psychiatry 2012;83: 681-6.

[14] Irani SR, Bera K, Waters P, et al. N-methyl-D-aspartate antibody encephalitis: temporal progression of clinical and paraclinical observations in a predominantly non-paraneoplastic disorder of both sexes. Brain 2010;133: $1655-67$.

[15] Vitaliani R, Mason W, Ances B, Zwerdling T, Jiang Z, Dalmau J. Paraneoplastic encephalitis, psychiatric symptoms, and hypoventilation in ovarian teratoma. Ann Neurol 2005;58:594-604.

[16] Irani SR, Michell AW, Lang B, et al. Faciobrachial dystonic seizures precede Lgi1 antibody limbic encephalitis. Ann Neurol 2011;69:892-900.

[17] Shin YW, Lee ST, Shin JW, et al. VGKC-complex/LGI1-antibody encephalitis: clinical manifestations and response to immunotherapy. J Neuroimmunol 2013;265:75-81.

[18] Masangkay N, Basu S, Moghbel M, Kwee T, Alavi A. Brain 18F-FDGPET characteristics in patients with paraneoplastic neurological syndrome and its correlation with clinical and MRI findings. Nucl Med Commun 2014;35:1038-46.

[19] Baumgartner A, Rauer S, Mader I, Meyer PT. Cerebral FDG-PET and MRI findings in autoimmune limbic encephalitis: correlation with autoantibody types. J Neurol 2013;260:2744-53.

[20] Ances BM, Vitaliani R, Taylor RA, et al. Treatment-responsive limbic encephalitis identified by neuropil antibodies: MRI and PET correlates. Brain 2005;128:1764-77.

[21] Pillai SC, Gill D, Webster R, Howman-Giles R, Dale RC. Cortical hypometabolism demonstrated by PET in relapsing NMDA receptor encephalitis. Pediatr Neurol 2010;43:217-20.

[22] Titulaer MJ, Soffietti R, Dalmau J, et al. Screening for tumours in paraneoplastic syndromes: report of an EFNS task force. Eur J Neurol 2011;18 [19-e13]. 
[23] Lee SW, Park MC, Lee SK, Park YB. The efficacy of brain (18)Ffluorodeoxyglucose positron emission tomography in neuropsychiatric lupus patients with normal brain magnetic resonance imaging findings. Lupus 2012;21:1531-7.

[24] Peterson PL, Axford JS, Isenberg D. Imaging in CNS lupus. Best Pract Res Clin Rheumatol 2005;19:727-39.

[25] Joseph FG, Scolding NJ. Neurolupus. Pract Neurol 2010;10:4-15.

[26] Fiorella DJ, Provenzale JM, Coleman RE, Crain BJ, Al-Sugair AA. (18)Ffluorodeoxyglucose positron emission tomography and MR imaging findings in Rasmussen encephalitis. AJNR Am J Neuroradiol 2001;22:1291-9.
[27] Varadkar S, Bien CG, Kruse CA, et al. Rasmussen's encephalitis: clinical features, pathobiology, and treatment advances. Lancet Neurol 2014;13: 195-205.

[28] Leypoldt F, Wandinger KP. Paraneoplastic neurological syndromes. Clin Exp Immunol 2014;175:336-48.

[29] Graus F, Dalmau J. Paraneoplastic neurological syndromes. Curr Opin Neurol 2012;25:795-801.

[30] Vincent A, Bien CG, Irani SR, Waters P. Autoantibodies associated with diseases of the CNS: new developments and future challenges. Lancet Neurol 2011;10:759-72.

Pour citer cet article : Lagarde S, Guedj E. Apport de la tomographie par émission de positons (TEP) cérébrale dans les encéphalites dysimmunitaires. Médecine Nucléaire (2015), http://dx.doi.org/10.1016/j.mednuc.2015.03.002 